\title{
On the influence of noise on the critical and oscillatory behavior of a predator-prey model: coherent stochastic resonance at the proper frequency of the system
}

\author{
A.F. Rozenfeld ${ }^{\text {a }}$, C.J. Tessone ${ }^{\text {b,c }}$, E. Albano ${ }^{\text {a }}$, H.S. Wio ${ }^{\text {b,* }}$ \\ a Instituto de Investigaciones Fisicoquímicas Teóricas y Aplicadas (INIFTA), UNLP, CONICET, CIC. Suc 4, C.C. 16, 1900 La Plata, Argentina \\ ${ }^{\mathrm{b}}$ Centro Atómico Bariloche (CNEA) and Instituto Balseiro (CNEA and UNC), 8400 San Carlos de Bariloche, Argentina \\ c Instituto de Física de La Plata, C.C. 63, Universidad Nacional de La Plata, 1900 La Plata, Argentina \\ Received 12 April 2000; received in revised form 1 January 2001; accepted 3 January 2001 \\ Communicated by C.R. Doering
}

\begin{abstract}
Noise-induced changes in the critical and oscillatory behavior of a prey-predator system are studied using power spectrum density and spectral amplification factor (SAF) analysis. In the absence of external noise, the population densities exhibit three kinds of asymptotic behavior, namely: absorbing state, fixed point (FP) and an oscillatory regime with a well defined proper (natural) frequency. The addition of noise destabilizes the FP phase inducing a transition to a new OR. Surprisingly, it is found that when a periodic signal is added to the control parameter, the system responds robustly, without relevant changes in its behavior. Nevertheless, the coherent stochastic resonance phenomenon is found only at the proper frequency. Also, a method based on SAF allows us to locate very accurately the transition points between the different regimes. @ 2001 Elsevier Science B.V. All rights reserved.
\end{abstract}

Oscillatory behavior is ubiquitous in all aspects of Nature. For instance, time periodic patterns can be found in problems involving the relation between different species (competition, predator-prey, coexistence, etc.) [1,2]. Oscillatory behavior can be also found in spatial dispersion and competition of living or chemical species [2-6], and even in some physiological situations (cardiac and circadian rhythms) [7].

Recently, and related to the interplay between oscillations and noise, the phenomenon of stochastic res-

\footnotetext{
* Corresponding author.

E-mail addresses: aleroz@inifta.unlp.edu.ar (A.F. Rozenfeld), tessonec@venus.fisica.unlp.edu.ar (C.J. Tessone), ealbano@inifta.unlp.edu.ar (E. Albano),wio@cab.cnea.gov.ar (H.S. Wio).
}

onance (SR) has been studied in different physical, chemical and biological contexts [8]. In particular, SR has been found to play a relevant role in several problems in biology: mammalian sensory systems, increment of the tactile capacity, visual perception, effects of low frequency and low amplitude electromagnetic fields, etc. [9]. Even the problem of coupling among SR units has been studied [10,11].

A related, albeit slightly different phenomenon, is the so-called stochastic coherent resonance (SCR) [12]. This phenomenon, that resembles SR, corresponds to a situation where the system shows noiseinduced coherent oscillations [13] without an externally applied signal or a discrete component in its spectrum (in this aspect it differs from SR without periodic forcing as discussed in [14]). Here we analyze 
the effect of fluctuations on a lattice gas model for a prey-predator system with smart pursuit and escape. Our aim is to analyze the possibility that a SCR-like phenomenon can occur in such a system.

Our lattice gas model, which is a variant of the cellular automata proposed by Boccara et al. [15], is defined as follows: a lattice site can be either empty or occupied either by a prey or a predator. Double occupancy of lattice sites is forbidden. The system evolves according to consecutive cycles: (i) competition of species and (ii) escape-pursuit dynamics.

The competition rules are as follows: (a) Preys have an offspring occupying an empty next neighbor site with probability $B_{P H}$ (birth probability of preys) in case of absence of predators within their $V_{R H}$ (visual range of preys). (b) Predators can eat a prey that is in their $M_{R P}$ (movement range of predators) with probability $D_{P H}$ (death probability of preys). (c) Predators who have already eaten a prey can produce an offspring in the site occupied previously by the eaten prey, with probability $B_{P P}$ (birth probability of predators).(d) Predators can suddenly die with probability $D_{P P}$ (death probability of predators).

The rules for an escape-pursuit process are as follows: (a) Preys calculate the gradient of the density of predators in their $V_{R H}$ and move into an empty site in the opposite direction. (b) Predators calculate the gradient of the density of preys in their $V_{R P}$ (visual range of predators) and move into an empty site in that direction.

We have restricted ourselves to investigating the dependence of the system on the predator birth probability $\left(B_{P P}\right)$, while the remaining parameters are kept constant, namely: $M_{R P}=V_{R P}=V_{R H}=1, D_{P P}=$ $0.25, D_{P H}=1$, and $B_{P H}=0.5$. The model is studied within a mean field approach. Further details on the model and results of Monte Carlo simulations have already been published [16].

With the above considerations, the mean field equations for the system can be derived evaluating the rates of all processes which may change the species densities. Then, it is obtained

$$
\begin{aligned}
\partial_{t} \rho_{P}=\rho_{P}(B-D), \\
\partial_{t} \rho_{H}=\rho_{H}\{A-(B+C) \\
\left.\quad \times\left[1-\left(1-\rho_{P}\right)^{\left(2 M_{R P}+1\right)^{2}-1}\right]\right\},
\end{aligned}
$$

where $\rho_{P}$ and $\rho_{H}$ are the global predator and prey densities, respectively. Also the rates are given by: (i) The rate of prey reproduction $A=B_{P H}(1-$ $\left.D_{P P}\right)^{\left(2 V_{R H}+1\right)^{2}-1}\left[1-\left(\rho_{P}+\rho_{H}\right)^{8}\right]$ which comprises the probability of a prey to have an offspring $\left(B_{P H}\right)$ in a neighboring empty state (first bracket) provided the absence of predators in the neighborhood of the progenitor (second bracket). (ii) The rate of predator reproduction $B=\left(1-D_{P P}\right) B_{P P} D_{P H}[1-(1-$ $\left.\rho_{H}\right)^{\left(2 M_{R P}+1\right)^{2}-1}$, which comprises the probability of the predator to survive $\left(1-D_{P P}\right)$, the birth probability of predator $\left(B_{P P}\right)$, the death probability of a prey $\left(D_{P H}\right)$ and the probability of existence of a prey in the visual range of a predator (last term). (iii) The probability of a predator to catch a prey $C=\left(1-D_{P P}\right)(1-$ $\left.B_{P P}\right) D_{P H}\left[1-\left(1-\rho_{H}\right)^{\left(2 M_{R P}+1\right)^{2}-1}\right]$ is equal to the previous term $B$, except for the fact that the predator does not have an offspring and consequently $B_{P P}$ has been replaced by $\left(1-B_{P P}\right)$. (iv) Finally, the dying probability of predators is $D=D_{P P}$.

In order to study the influence of external perturbations on the system, it is assumed that the control parameter, $B_{P P}$, is time dependent and has the form

$B_{P P}(t)=B_{P P o}+Q \xi(t)+\varepsilon \cos \left(\Omega_{\varepsilon} t\right)$,

where $\xi(t)$ is a normalized Gaussian white noise of intensity $Q$ and $\varepsilon$ is the amplitude of a periodic external signal with frequency $\Omega_{\varepsilon}$. In any biological system a parameter such as the birth probability of predators (or any other) will not be constant in time. Effectively, the environment competition, climate variability, etc., will alter the characteristics of the birth rate of all species. Although it is true that all the parameters of this system will change in time, as a first step in our study, we simplify the analysis by only considering modifications on the $B_{P P}$ parameter.

Clearly, the choice of a Gaussian noise implies the possibility of having negative values of $B_{P P}(t)$. Such a case has no physical meaning but, as the width of the Gaussian distribution is too small, the small negative tail does not affect our results.

Initially, the behavior of the system has been studied in the absence of any external perturbation, i.e., fixed $B_{P P}$. It was found that the densities of species, $\rho_{P}$ and $\rho_{H}$ show the following asymptotic behavior depending on the value of $B_{P P}$ : (i) If $B_{P P}<1 / 3$ the system evolves toward the extinction of predators, 


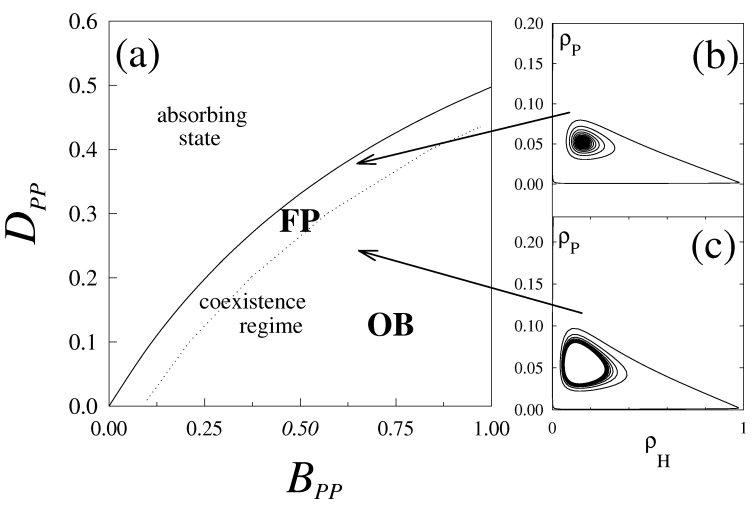

Fig. 1. Phase diagram of the prey-predator system as obtained solving the mean-field equations in absence of noise. (a) Plot of $D_{P P}$ versus $B_{P P}$ showing the critical curve (solid line) for the irreversible phase transition between the prey-predator coexistence regime and the absorbing state where predators become extinct. The dotted line shows the critical curve for the transition between the fixed point (FP) regime and the oscillatory behavior (OB). (b) and (c) show the dependence of the population densities for the different regimes of the system, namely FP and OB, respectively.

namely $\rho_{P}=0$ and $\rho_{H}=1$. Such a state is an "absorbing regime" (AR), because the system cannot escape from it. (ii) If $B_{P P} \geqslant 1 / 3$ the final state of the system is a steady regime with coexistence of prey and predators. However, within this regime, dynamic behavior changes according the value of $B_{P P}$. Thus, if $B_{P P}$ is near the AR phase, $\rho_{P}$ and $\rho_{H}$ reach constant values, say a "fixed point" (FP) regime (see Fig. 1). On the other hand, for high enough values of $B_{P P}$, the system enters an "oscillatory regime" (OR), since both populations exhibit self-sustained oscillations (Fig. 1). It should be noted that the proper frequency of the system is independent of $B_{P P}$.

Hence, $B_{P P}$ is the control parameter governing the system dynamics, while $\rho_{P}$ and $\rho_{H}$ are order parameters. Then, it is possible to infer that there are critical values $B_{P P}^{1 c}$ and $B_{P P}^{2 c}$ separating both $\mathrm{AR}$ and $\mathrm{FP}$, and FP and OR, respectively.

The discrete versions of Eqs. (1) and (2) have been solved in order to: (i) determine if the system shows the SCR phenomenon; (ii) provide methods for the accurate localization of the critical values.

Time series of population densities, $\rho_{H}(t)$ and $\rho_{H}(t)$, were computed recursively from the discrete mean field equations. Then, the respective temporal self-correlation functions $K_{H}(\tau)$ and $K_{P}(\tau)$, given by

$$
\begin{aligned}
& K_{H}(\tau)=\left\langle\rho_{H}(t) \rho_{H}(t+\tau)\right\rangle_{t}, \\
& K_{P}(\tau)=\left\langle\rho_{P}(t) \rho_{P}(t+\tau)\right\rangle_{t},
\end{aligned}
$$

were calculated. Here \langle\rangle$_{t}$ represents the time average. It is well known that the power spectrum density (psd) is just the Fourier Transform of such functions $S_{H}(\omega)=\mathcal{F}\left[K_{H}(\tau)\right]$ and $S_{P}(\omega)=\mathcal{F}\left[K_{P}(\tau)\right]$, respectively. Then, the SNR is readily obtained as

$$
R_{H}=10 \log _{10}\left(\frac{S_{H}^{s}}{S_{H}^{n}}\right), \quad R_{P}=10 \log _{10}\left(\frac{S_{P}^{s}}{S_{P}^{n}}\right),
$$

where $S_{H}^{S}\left(S_{P}^{S}\right)$ is the output power at the frequency of the signal considered, and $S_{H}^{n}\left(S_{P}^{n}\right)$ is the output power of the noisy background at the same frequency, both of them obtained from the prey (predator) timeseries, respectively. From now on, we will present the results for the predator time-series only, as the prey ones shows qualitatively the same behavior. Also, the subscripts will be omitted to simplify the notation.

It is worthwhile to remark here, that the analysis was made considering the proper frequency of the system, $\Omega^{*}=0.03$. At this frequency $R$ reaches a significant value, in contrast to its value at the forcing frequency $\left(\Omega_{\varepsilon}\right)$ where $R$ is negligible. We also verified that the periodic term in Eq. (3) does not introduce changes in the dynamics of the system. Thus, we will not consider in this Letter any oscillating perturbations on the control parameter.

Figs. 2 and 3 show the psd when the system is in the FP and OR regimes, respectively. Qualitatively, the results can be understood as follows (see Fig. 2): if the system is in FP, the psd at the proper frequency is zero when $Q=0$. For very low noise strengths $\left(Q<10^{-4}\right), S\left(\Omega^{*}\right)$ is an increasing function of $Q$. However, the situation changes when the noise is strong enough $\left(Q \sim 10^{-3}\right)$. Here, the peak at the proper frequency is hidden by the noisy background. Then, it is clear that the function $R(Q)$ would have a maximum in the considered range of $Q$. In contrast, within the OR (see Fig. 3) $S\left(\Omega^{*}\right)$ has a finite (nonzero) value in the limit $Q \rightarrow 0$. As the noise strength grows, such a peak decreases and broadens. Then, for this case the SNR of the system as a function of noise strength is expected to be a monotonic decreasing function of such a parameter. 

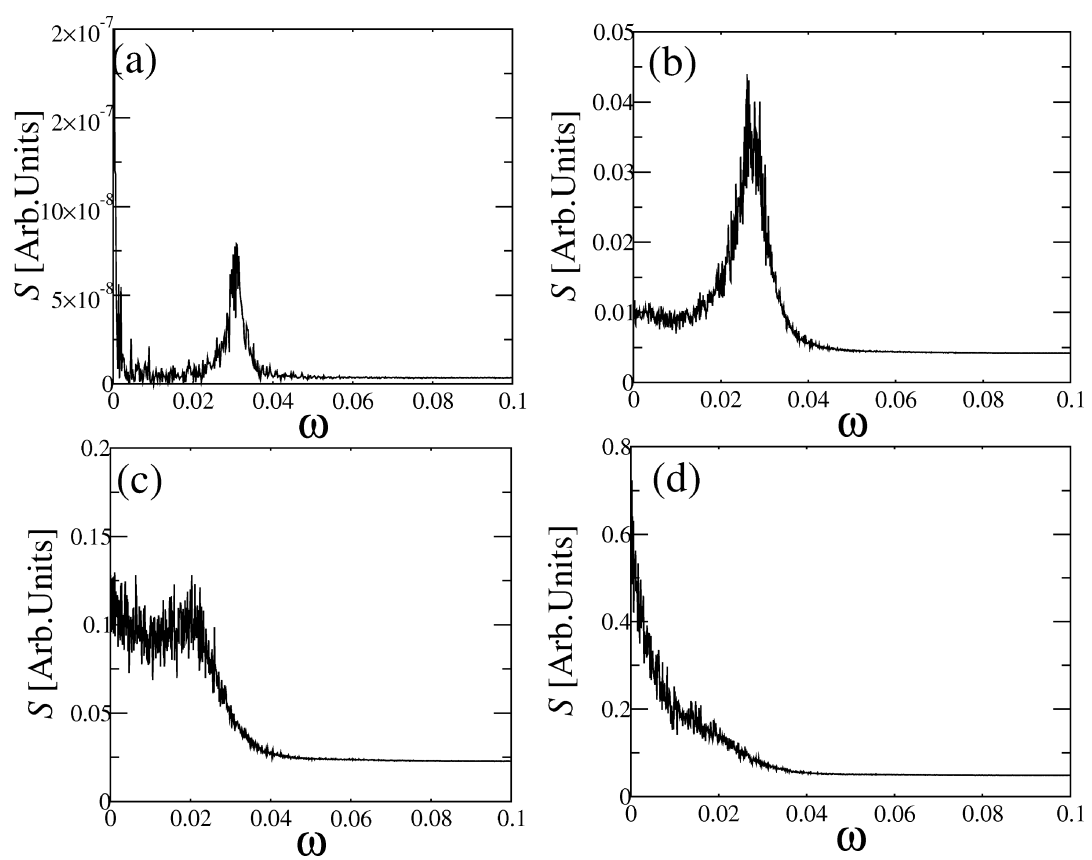

Fig. 2. Plots showing the dependence of the psd when the noise is modified. In absence of noise the system is in the fixed point regime since $B_{P P O}=0.42$ is kept constant. In each graph, we varied $Q$, the values being: (a) $Q=10^{-8}$, (b) $Q=10^{-2}$, (c) $Q=4.45 \times 10^{-2}$, (d) $Q=10^{-1}$.
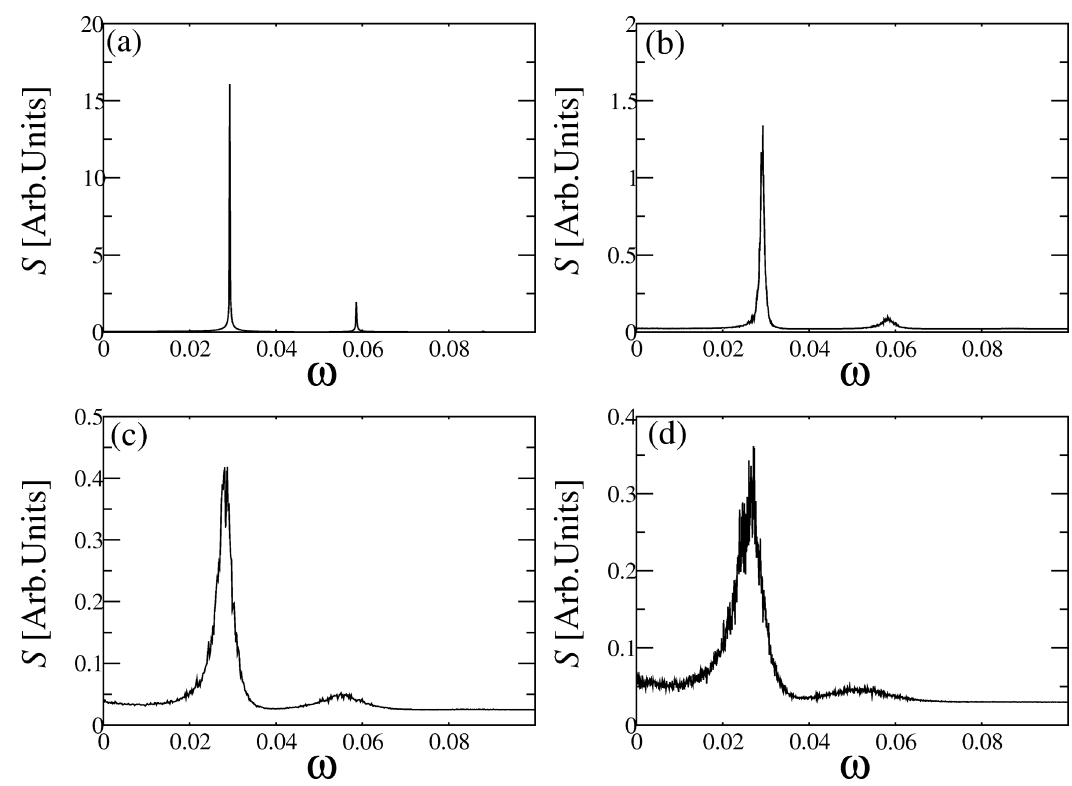

Fig. 3. Plots of $S$ versus $\omega$ corresponding to the time series of the predator density. In this case, when $Q=0$ the system is in OR. The results are for: (a) $Q=0$, (b) $Q=10^{-4}$, (c) $Q=10^{-2}$ and (d) $Q=10^{-1}$, keeping $B_{P P o}=0.70$ constant. 

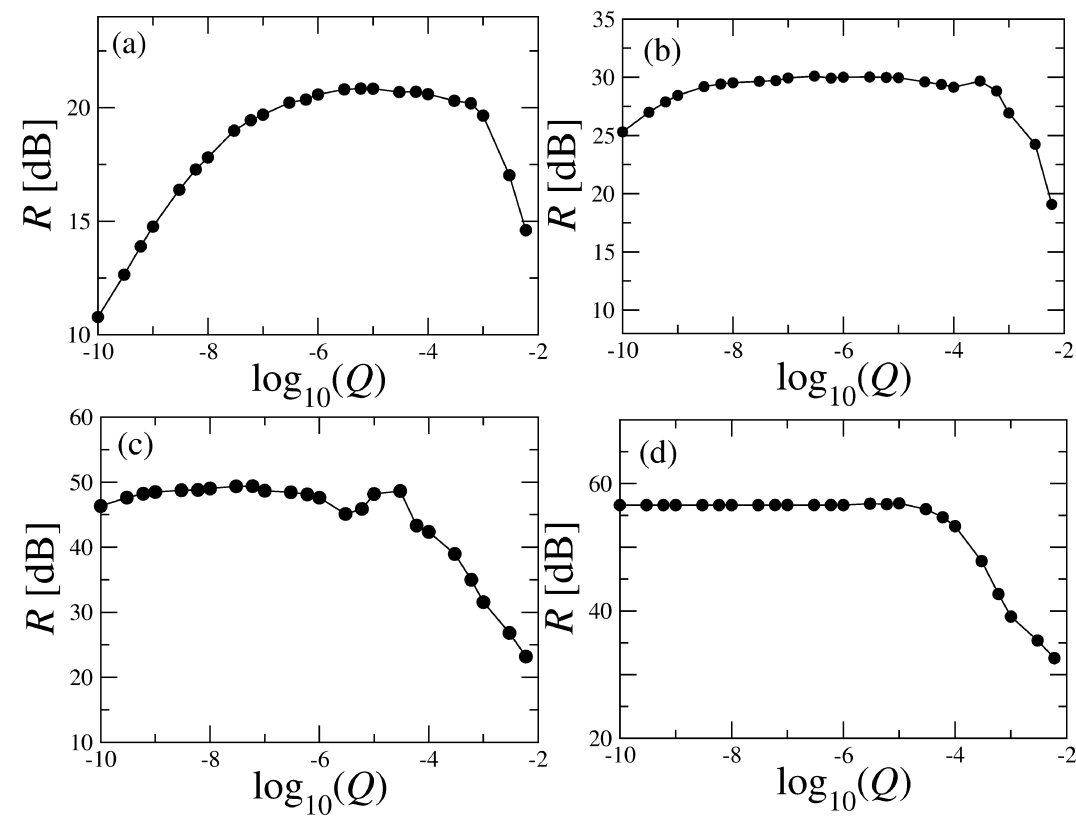

Fig. 4. Plots of the output SNR as a function of noise strength, $Q$, obtained for different values of $B_{P P_{0}}$. A SCR phenomenon is apparent when $B_{P P o}$ is within FP regime, but it disappears if the system is within OR. (a) $B_{P P o}=0.42$, (b) $Q=B_{P P o}=0.45$, (c) $B_{P P o}=0.47$, (d) $B_{P P O}=0.50$.

In the following, a method to locate the phase transition between FP and OR regimes, by means of the calculation of the SNR, is developed. Fig. 4 shows plots of the SNR as a function of $Q$ for different values of $B_{P P O}$. The SCR phenomenon is only apparent for values of $B_{P P o} \leqslant 0.47$. Such a dependence on $Q$ is the main characteristic of the system for the FP regime. When $B_{P P o}>0.47$ the SNR is a decreasing function of $Q$, which is the expected behavior when the system is in OR. The value of the parameter $B_{P P}^{2 c}$ where the SCR phenomenon disappears can readily be identified with the critical point. Hence, our best estimate for such a transition point is given by $B_{P P}^{2 c} \cong 0.47 \pm 0.01$.

Fig. 5 shows the dependence of SNR as a function of $B_{P P o}$, for some fixed values of $Q$. In the absence of noise the critical point can easily be obtained from the plot (Fig. 5(a)), yielding $B_{P P}^{c} \cong 0.47 \pm 0.01$, in agreement with the value obtained through the method discussed above.

A surprising consequence of the addition of noise is that, as can be seen in Figs. 5(b)-(e), even for control parameter values $B_{P P o}<0.47$ the system oscillates at the proper frequency. This indicates that the FP phase is unstable, and even a small perturbation drives it into an oscillatory behavior. Also, the plots of $R$ vs. $B_{P P_{0}}$ exhibit linear behavior for lower values of the parameter, e.g., $B_{P P O}<0.46$. Extrapolations by linear regression to the limit $R \rightarrow 0$ give $B_{P P}^{1 c} a \cong 0.330 \pm$ 0.005 which is the critical point for the irreversible transition from the coexistence regime to the AS. This figure is in excellent agreement with our previous estimate $B_{P P}^{1 c}=1 / 3$ obtained simply solving the mean field equations in the absence of noise, as discussed earlier.

Subsequently, for small noise values $Q \leqslant 10^{-4}$ (Figs. 5(b) and (c)), the onset of a shoulder is clearly observed, say for $B_{P P o}>0.47$. As will be discussed later, this can be associated to another phase transition between intrinsically different oscillatory regimes.

In the search of a method to characterize these two different oscillating phases we make use of the fact that, although this is an extremely nonlinear system, the time series $\rho_{H}$ and $\rho_{P}$ are composed only by a single frequency (the proper one) and its harmonics. Supported by this numerical evidence, it is assumed that for long times, the solution of Eqs. (1) and (2) 

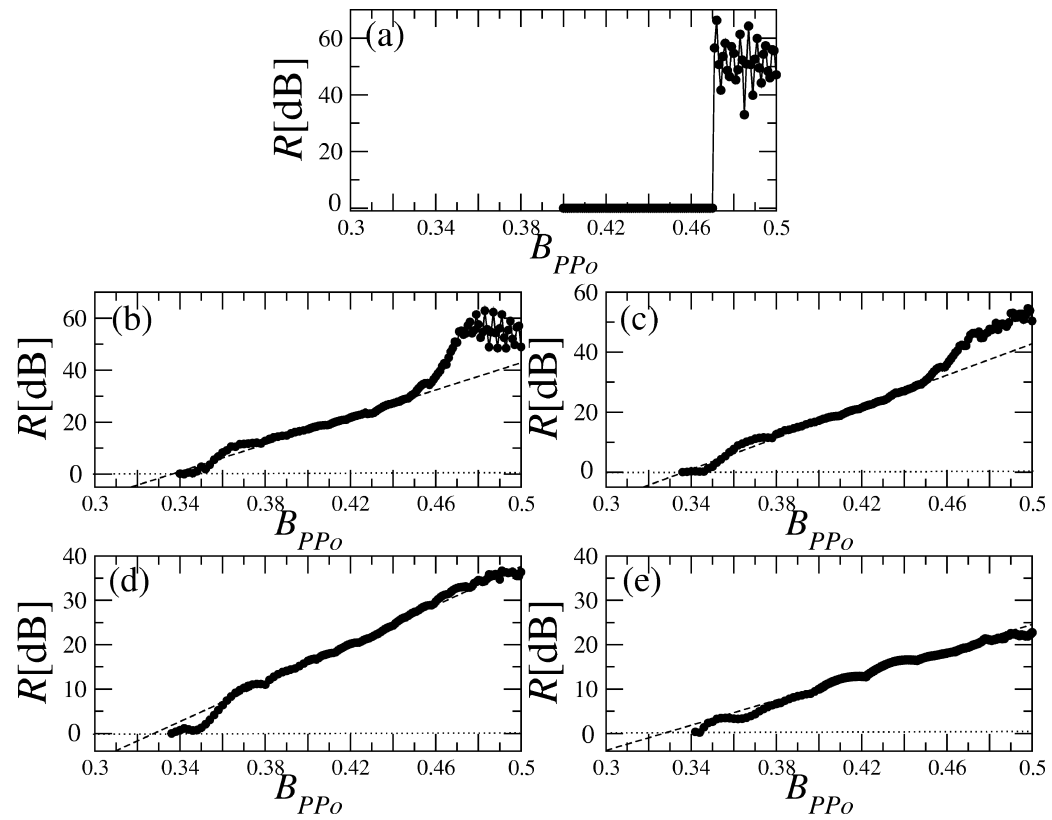

Fig. 5. Plots of $R$ as a function of the control parameter $B_{P P O}$. In each plot, the noise strength is kept constant, as follows: (a) $Q=0$, (b) $Q=10^{-5}$, (c) $Q=10^{-4}$, (d) $Q=10^{-3}$ and (e) $Q=10^{-2}$.

approaches a periodic function, which will be of Floquet type. Then, the corresponding stationary selfcorrelation function $\left(K_{\text {as }}(\tau)\right)$, can be decomposed in a Fourier series, i.e.,

$K(\tau)=\sum_{n=1}^{\infty} A_{n} \sin (n \Omega \tau)+\sum_{n=0}^{\infty} B_{n} \cos (n \Omega \tau)$.

It is expected that in the FP regime, all the coefficients (with the exception of $B_{0}$ ) will vanish. Also, in OR, at least the terms $A_{1}$ and $B_{1}$ (related with the amplitude of oscillations) must be non-zero. Hence, measuring the value of those coefficients as a function of $Q$ and $B_{P P}$ it may be possible to find the phase transition.

Once again, this is a SCR phenomenon, as analyzed within the framework used in Ref. [17], where the characterization of SR is done by means of the spectral amplification factor (SAF). Following this course, we define $W$, the degree of oscillation (this will indicate how much inside the OR is the system), as the inner product between $K_{a s}(\tau)$ and the first harmonic of Eq. (6), which gives

$W=A_{1}^{2}+B_{1}^{2}$.
Fig. 6 shows plots of $W$ as a function of the control parameter $B_{P P o}$, in the absence (filled circles) and presence (empty circles) of noise. It is easy to distinguish the following: (i) In the absence of noise the FP regime is clearly observed in the figure, and a value $B_{P P}^{2 c}=0.47$ is found, in excellent agreement with the results previously discussed in connection with the behavior of SNR vs. $B_{P P o}$ (Fig. 5). (ii) In the presence of noise it can be seen that the value of $W$ increases for $B_{P P O}<0.45$. Then, a new oscillatory regime is apparent where $W$ is independent of $B_{P P o}$, and such a behavior arises instead of the FP phase, because of the applied low-intensity noise. This phenomenon can be understood in terms of a noise-induced phase transition. On the other hand, the usual OR where $W$ monotonically increases with $B_{P P o}$, is obtained for $B_{P P o} \geqslant 0.45$. In fact, the value of $B_{P P o}$ for which the system enters into the usual OR, suffers a shift due to the presence of noise [18]. Effectively, it changes from $B_{P P_{O}}(Q=0)=0.47$ to $B_{P P_{o}}\left(Q=10^{-5}\right)=0.45$.

The former study of $W$ behavior allows us to give further support to our discussion of the results shown in Fig. 5. Subtracting the linear behavior, the follow- 


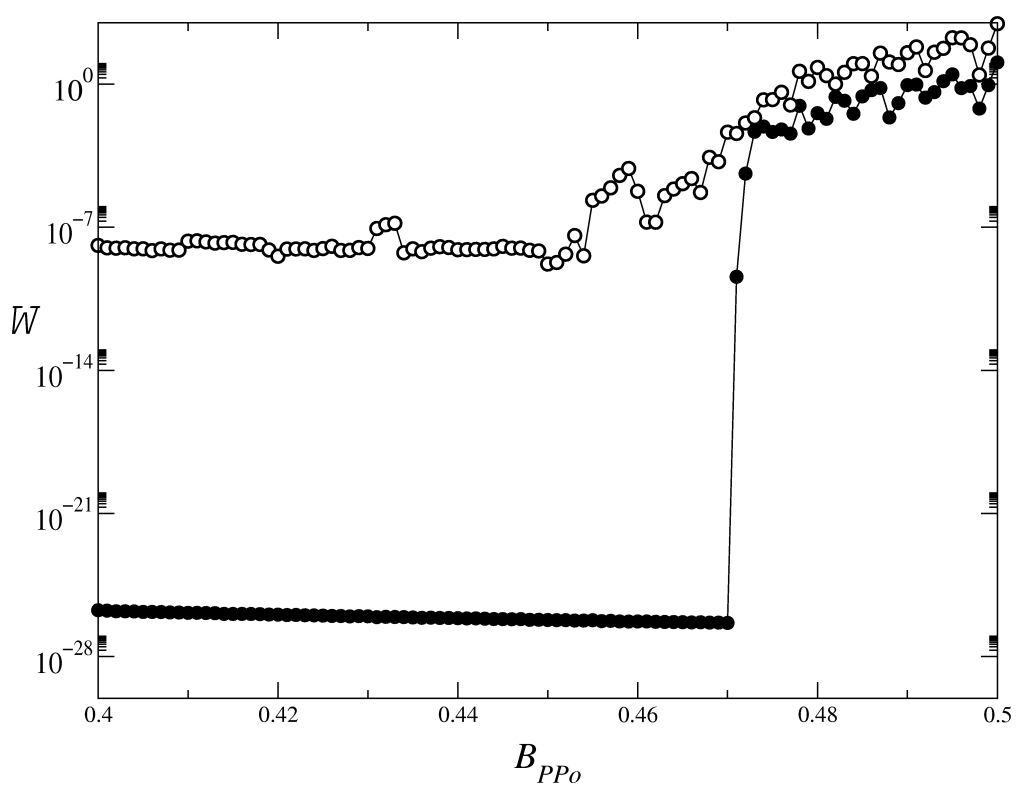

Fig. 6. Plots of the degree of oscillation as a function of $B_{P P o}$, obtained for $Q=0$ (a) and $Q=10^{-5}$ (b). In both cases, the phase transition between FP and OR can be observed. Notice that such a transition is shifted in the presence of a small noise strength.

ing estimates for the phase transition are obtained: $B_{P P_{0}}\left(Q=10^{-5}\right) \cong 0.461 \pm 0.005$ and $B_{P P_{o}}(Q=$ $\left.10^{-4}\right) \cong 0.453 \pm 0.005$. Finally, when $Q$ increases (Figs. 5(d) and (e)), the linear dependence on $B_{P P o}$ holds for a wider range in such a variable and consequently, the phase transition is more difficult to detect. This is because the system is strongly oscillatory even if (in absence of noise) $B_{P P O}$ is within the FP regime. In fact, the linear tendency in the function $R\left(B_{P P o}\right)$ observed for small values of $Q$ is broken when the parameter is set within the OR. Also, when the OR is masked by the response to a larger noise, the linear dependence is not broken but the critical point cannot be located anymore. Furthermore, from Figs. 5(a) and 6 one observes that in the absence of noise the transition FP $\leftrightarrow$ OR is abrupt resembling first-order behavior. However, when the noise is added the transition between the two oscillatory regimes not only becomes shifted, but also rounded (Figs. 5(b) and 6).

Summarizing, the effect caused by external noise on a prey-predator system, with smart pursuit evasion is studied by means of SNR and degree of oscillation techniques. In order to perform the study, the behavior of the system is analyzed when a small amount of white noise is applied over a control parameter $\left(B_{P P o}\right)$. It is found that if (in the absence of noise) such a parameter is in FP, a coherent stochastic resonance phenomenon arises. In fact, only the proper frequency becomes strongly enhanced, while any externally applied oscillatory signal is not amplified. Nevertheless, such an enhancement arises only for a (intermediate) range of noise strength $\left(Q \sim 10^{-6}\right)$ and it vanishes in two distinct limiting cases: (i) when noise is not applied, and (ii) when the noise is too large $\left(Q>10^{-3}\right)$. It should be remarked that this resonant phenomenon disappears if the system is driven to the OR, tuning the control parameter. Then, it is possible to locate, looking at the dependence of SNR as a function of noise, the critical point at which the phase transition between the FP and the OR occurs.

In the presence of noise, the FP phase is replaced by a new oscillatory regime where the amplitude of oscillations remains constant, allowing us to assert that the FP phase is less stable than the OR one. The dependence of SNR as a function of $B_{P P O}$ shows linear behavior and the extrapolation of such a line intersects the SNR axis at $B_{P P o}=0.330 \pm 0.005$. This value is the critical point where the irreversible phase 
transition to the AS occurs. Thus, this method allows us to find both phase transitions: (i) the irreversible one between the coexistence regime and the AS, and in the absence of noise (ii) the reversible one between the FP and the OR regimes, respectively.

We have also defined the degree of oscillation as the corresponding terms of the first harmonic in the Floquet-type asymptotic expansion of the selfcorrelation function of the population time series. This characterization, provides us with an alternative method to locate the phase transition between FP and OR.

Furthermore, the abrupt (first-order like) transition between the FP and the OR in absence of noise becomes clearly rounded (second-order like) when noise is applied. These results point out that the effect of noise is not merely restricted to the shift of the critical point, but instead that the whole nature of the transition changes.

We expect that the findings reported in this Letter will contribute to the understanding of resonant effects and critical behavior in actual competitive population systems, since in nature they are exposed to different sources of external noise.

\section{Acknowledgements}

The authors want to thank V. Grünfeld for a critical reading of the manuscript.

\section{References}

[1] V. Volterra, R. Comitato Talassografico Italiano Mem. 131 (1927) 1 ;

M. Peschel, W. Mende, The Predator-Prey Model: Do We Live in a Volterra World?, Springer, Wien, 1986.

[2] J.D. Murray, Mathematical Biology, Springer, New York, 1989.
[3] A. Okubo, Diffusion and Ecological Problems: Mathematical Models, Biomathematical Series, Vol. 10, Springer, New York, 1980.

[4] P.L. Chow, W.C. Tam, Bull. Math. Biol. 38 (1976) 643.

[5] P.C. Fife, Mathematical Aspects of Reacting and Diffusing Systems, Springer, New York, 1979.

[6] G. Nicolis, I. Prigogine, Self Organization in Nonequilibrium Systems, Wiley, New York, 1977.

[7] A.T. Winfree, The Geometry of Biological Time, Lecture Notes in Biomath., Vol. 8, Springer, Berlin, 1980.

[8] K. Wiesenfeld, F. Moss, Nature 373 (1995) 33; L. Gammaitoni, P. Hänggi, P. Jung, F. Marchesoni, Rev. Mod. Phys. 70 (1998) 223.

[9] J.K. Douglas, L. Wilkens, E. Pantazelou, F. Moss, Nature 365 (1993) 337;

S.M. Bezrukov, I. Vodyanoy, Nature 378 (1995) 362;

J.J. Collins, T.T. Imhoff, P. Grigg, Nature 383 (1996) 770;

I.L. Kruglikov, H. Dertinger, Bioelectromag. 15 (1994) 539;

B.J. Gluckman et al., Phys. Rev. Lett. 77 (1996) 4098;

E. Simonotto et al., Phys. Rev. Lett. 78 (1997) 1186.

[10] J.F. Lindner, B.K. Meadows, W.L. Ditto, M.E. Inchiosa, A.R. Bulsara, Phys. Rev. Lett. 75 (1995) 3;

J.F. Lindner, B.K. Meadows, W.L. Ditto, M.E. Inchiosa, A.R. Bulsara, Phys. Rev. E 53 (1996) 2081.

[11] F. Castelpoggi, H.S. Wio, Europhys. Lett. 38 (1997) 91; F. Castelpoggi, H.S. Wio, Phys. Rev. E 57 (1998) 5112; M. Kuperman, H.S. Wio, G. Izús, R. Deza, Phys. Rev. E 57 (1998) 5122;

M. Kuperman, H.S. Wio, G. Izús, R. Deza, F. Castelpoggi, Physica A 257 (1998) 275;

S. Bouzat, H.S. Wio, Phys. Rev. E 59 (1999) 5142.

[12] A. Pirovsky, J. Kurths, Phys. Rev. Lett. 78 (1997) 775; J.J. Casado, Phys. Lett. A 235 (1997) 489;

H. Hempel, L. Schimansky-Geier, J. Garcia-Ojalvo, Phys. Rev. Lett. 82 (1999) 3713.

[13] S. Zhong, H.W. Xin, J. Phys. Chem. A 104 (2000) 297, and references therein.

[14] H. Gang, T. Ditzinger, C.Z. Ning, H. Haken, Phys. Rev. Lett. 71 (1993) 807.

[15] N. Boccara, O. Robin, M. Roger, Phys. Rev. E 50 (1994) 4531.

[16] A. Rozenfeld, E. Albano, Physica A 266 (1999) 322.

[17] P. Jung, P. Hänggi, Europhys. Lett. 8 (1989) 505; C. Jüng, P. Hänggi, Phys. Rev. A 44 (1991) 8032.

[18] W. Horsthemke, R. Lefever, Noise-Induced Transitions: Theory and Applications in Physics, Chemistry and Biology, Springer, New York, 1984. 